\title{
EVANESCENT MICROWAVE PROBES USING COPLANAR WAVEGUIDE AND STRIPLINE FOR SUPER-RESOLUTION IMAGING OF MATERIALS
}

\author{
G. E. Ponchak' ${ }^{1}$, D. Akinwande' ${ }^{2}$, R. Ciocan', S. R. LeClair ${ }^{3}$, and M. Tabib-Azar ${ }^{2}$
}

1. NASA Lewis Research Center, 21000 Brookpark Rd., MS 54/5, Cleveland, OH 44135

2. Dept. of Electrical Engineering and Applied Physics, Case Western Reserve University, Cleveland, OH 44106

3. Air Force Research Laboratory, Materials \& Manufacturing Directorate, 2977 P. Street, Suite 13, Wright-Patterson AFB, OH

\section{ABSTRACT}

An evanescent field microwave imaging probe based on half-wavelength, microwave transmission line resonators is described. Optimization of the probe tip design, the coupling gap, and the data analysis has resulted in images of metal lines on semiconductor substrates with $2.6 \mu \mathrm{m}$ spatial resolution and a minimum detectable line width of $0.4 \mu \mathrm{m}$ at 1 $\mathrm{GHz}$.

\section{INTRODUCTION}

Non-destructive methods of imaging surface and subsurface structures and material properties are critical for acceptance testing and failure detection of countless applications including: semiconductor defect detection, thin film resistivity measurement, continuity of embedded transmission lines in high density interconnect multichip modules, and substrate epoxy void detection. For ultra-high resolution material property imaging, atomic force microscopy (AFM) [1] and scanning tunneling microscopy (STM) [2] have yielded atomic scale spatial resolution. Alternatively, optical and scanning electron microscopes are used for imaging surface structures with micron and submicron resolution. Each of these instruments though is expensive, labor intensive, and not readily incorporated into automated processing or manufacturing lines. Furthermore, they are not suitable for large sample size imaging.
Microwave microscopy using "nearfields" or evanescent fields has been used for imaging with a resolution of $\lambda_{\mathrm{o}} / 1000$ or better where $\lambda_{c}$ is the free space wavelength [3-9]. Evanescent field probes have used: open resonators with an aperture in one plane [3], rectangular waveguide with an aperture in the end plane $[4,5]$, the center conductor of coaxial transmission line [6,7], a coaxial transmission line resonator with an aperture [8], and microstrip resonators incorporating wire probes or loops $[5,9]$. For each of these probes, the dimensions of the aperture or wire that is used determine the resolution.

In this paper, we report the results of Evanescent Microwave Probes (EMPs) that use planar microwave transmission line resonators. Because these resonators are easily manufactured in linear arrays, quick characterization of large samples is possible. Furthermore, by optimizing the resonator coupling and tip design, the microwave microscope design, and the data analysis, a spatial resolution of $2.6 \mu \mathrm{m}$ using a $1 \mathrm{GHz}$ EMP is possible as demonstrated here for the first time.

\section{PROBE DESIGN}

Evanescent Microwave Probes use a microwave resonator as the source of evanescent fields and as a detector for perturbations to those fields. When planar microwave transmission lines such as 
microstrip, coplanar waveguide, and stripline are used, the open or short circuit terminated end of an end coupled resonator serve as the probe tip as shown in Figure 1. The electromagnetic fields extend outward from the probe tip a short distance. Thus, an object brought in close proximity to the tip will interact with the evanescent fields and change the loading of the resonator. For example, a perfect dielectric sample increases the probe capacitance and results in a lower resonant frequency of the same $Q$ as the unperturbed probe. This is demonstrated in Figure 2 which presents the reflection coefficient of a channelized coplanar waveguide (CCPW) [10] EMP when a high resistivity silicon wafer is placed near the probe tip. Metallic samples also increase the resonator end effect capacitance and lower the resonant frequency in a similar manner. Besides changing the resonant frequency, imperfect metal and insulating samples decrease the $Q$ of the resonator. By detecting these variations in the resonant frequency, $f_{0}$, and reflection coefficient magnitude, $\left|S_{11}\right|$, of the resonator, a wide range of material properties may be measured. The authors have measured variations in carrier concentration, carrier mobility, interface trap defects, cracks, voids, and material thickness variations with an EMP.

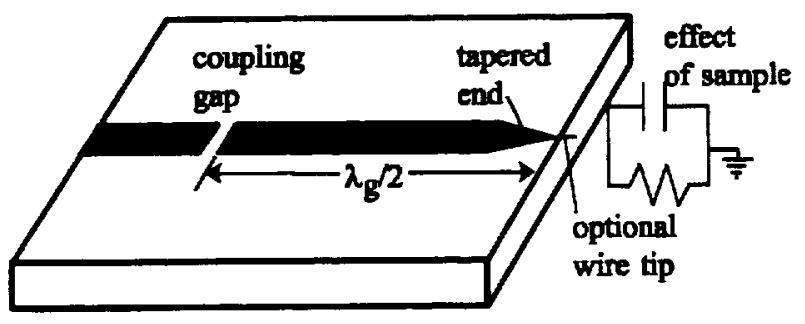

Figure 1: Schematic of microstrip resonator as used in an evanescent microwave probe.

To increase the spatial resolution of the probe, the end is tapered to a point as illustrated in Figure 1. Further improvement of the resolution is achieved by attaching a short, thin wire to the end of the probe tip. This is illustrated in Figure 1. The $Q$ of the resonator also strongly influences the sensitivity and resolution of the probe. Thus, it is important to optimize the coupling gap to achieve critical coupling. For our probes, critical coupling is achieved for the isolated probe or when no sample is present.

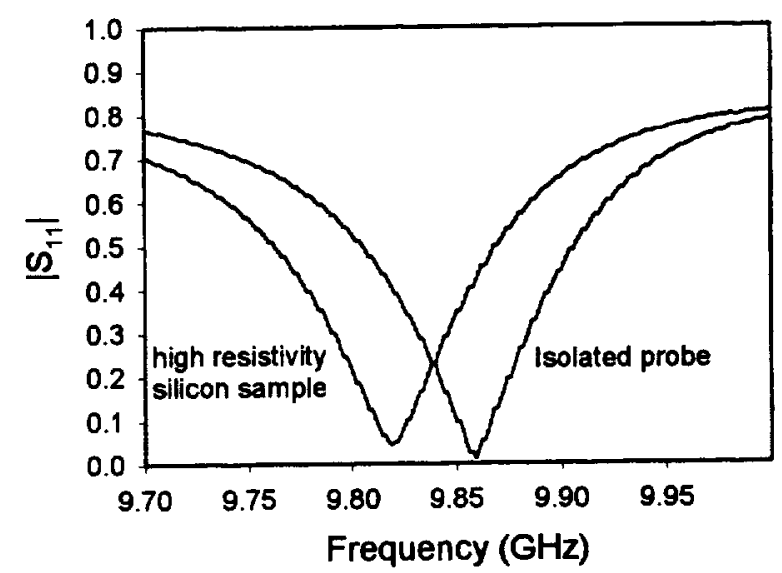

Figure 2: Measured |S11| of channelized coplanar waveguide probe both with and without high resistivity silicon sample near the probe tip.

In this paper, two types of resonators are described. The first uses a CCPW fabricated on a $0.3175 \mathrm{~cm}$ thick RT Duroid $\left(\varepsilon_{\mathrm{r}}=2.2\right)$ substrate. The center conductor width is $0.127 \mathrm{~cm}$ and the slot width is $0.028 \mathrm{~cm}$ except at the probe tip which is tapered to a point. Copper foil is used to connect the upper and lower ground planes to form the channelized CPW. The second probe is a stripline resonator fabricated on $\varepsilon_{\mathrm{r}}=3.8$ $\mathrm{RT} /$ Duroid with a strip width of $0.5 \mathrm{~cm}$. Both probes have a $20 \mu \mathrm{m}$ diameter wire tip at the end and are mounted in an aluminum fixture to eliminate coupling to other probes and decrease the background noise that would decrease the probe sensitivity. 


\section{MICROWAVE MICROSCOPE DESIGN}

The schematic of the microwave microscope is shown in Figure 3 [9]. The resonator and feedline is connected to a semirigid coaxial cable which is fed by a $0.5-12.5$ $\mathrm{GHz}$ signal generator through a circulator. A crystal microwave detector connected to the third port of the circulator produces a DC voltage proportional to the magnitude of the reflected signal. Samples are mounted on the $x$ $y$ stage platform and are vibrated in the $\mathrm{z}$ direction by a solenoid at approximately 100 $\mathrm{Hz}$. This enables synchronous detection of the signal by a lock-in amplifier to increase the signal-to-noise ratio. A Lab View program controls the positioning of the sample in three directions as well as measuring and monitoring the output voltage of the lock-in amplifier at each position of the sample.

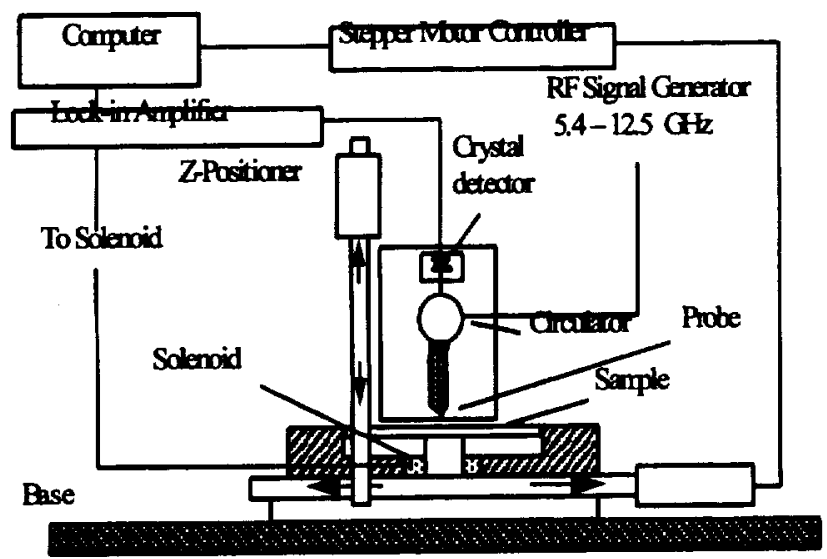

Figure 3: Schematic of the microwave microscope.

\section{RESULTS}

EMPs are characterized by determining the spatial resolution and minimum detectable sample. The spatial resolution is typically determined by imaging a small sample and reporting the full width of the EMP image at one half of the maximum signal level [6].

To characterize the CCPW, a $20 \mu \mathrm{m}$ diameter wire resting on a glass plate is imaged along the $x$ and $y$-axis where the $z$-axis is longitudinal with the CCPW resonator, the $x$ axis is normal to the plane of the probe substrate, and the $y$ axis is along the edge of the probe (see inset of Figure 4). The scanned image of the wire along the $x$ and $y$-axis for the CCPW EMP at $10 \mathrm{GHz}$ is shown in Figure 4. Using the definition previously given, the resolution is $25.5 \mu \mathrm{m}$ and $22 \mu \mathrm{m}$ along the $\mathrm{x}$ and $y$-axis respectively. The asymmetry of the $\mathrm{x}$-axis scan is believed to be due to the influence of the substrate. Note that for the $y$-axis scan, the substrate and wire are in the same relative position to each other throughout the scan.

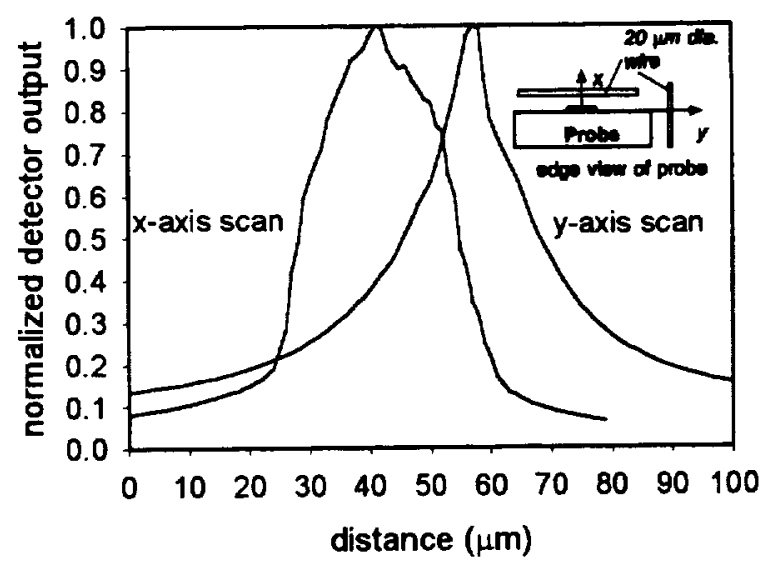

Figure 4: Scan of a $20 \mu \mathrm{m}$ diameter wire along the $x$ and $y$-axis using CCPW evanescent microwave probe.

The stripline EMP at $1 \mathrm{GHz}$ is used to image a $2 \mu \mathrm{m}$ wide straight metal trace on a CMOS grade Si substrate. An optical image, an EMP image, and an STM image of the sample are shown in Figures $5 \mathrm{a}, \mathrm{b}$ and $\mathrm{c}$ respectively. From this data, the resolution of the stripline EMP is determined to be $2.6 \mu \mathrm{m}$ and the minimum detectable line width is $0.4 \mu \mathrm{m}$.

\section{CONCLUSIONS}

A new EMP probe using a channelized coplanar waveguide resonator is described for the first time and demonstrated to have a resolution of $23.7 \mu \mathrm{m}$. By fully optimizing a stripline probe, an EMP at $1 \mathrm{GHz}$ has 
demonstrated $2.6 \mu \mathrm{m}$ resolution and $0.4 \mu \mathrm{m}$ minimum detectable line width.

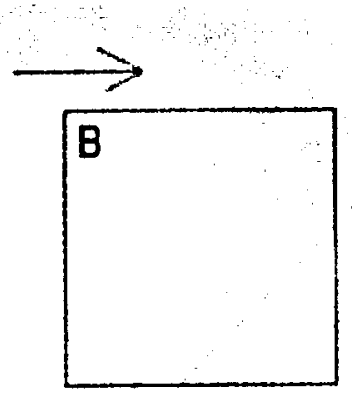

(a)

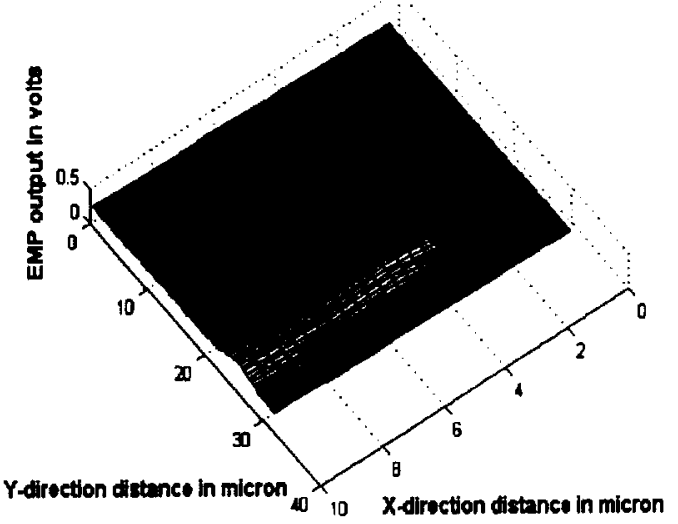

(b)

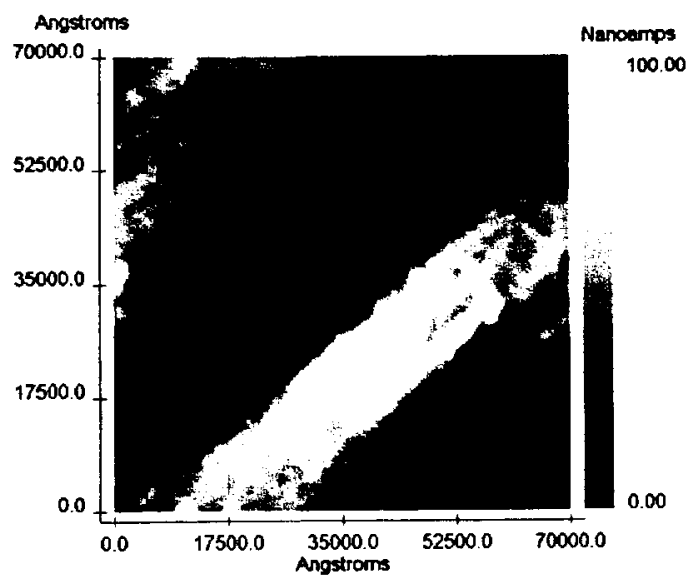

(c)

Figure 5: (a) optical image, (b) EMP image, and (c) STM image of $2 \mu \mathrm{m}$ wide metal trace on Si substrate.

\section{REFERENCES}

1. D. Sarid, "Scanning Force Microscopy," Oxford University Press, New York, 1991, pp. 5564.

2. U. Memmert, U. Hodel, and U. Hartmann, "Combined ultrahigh vacuum scanning tunneling microscope scanning electron microscope system," Rev. Sci. Instrum., 67(6), June 1996, pp. 22692273.

3. E. A. Ash and G. Nicholls, "Super-resolution aperture scanning microscope," Nature, Vol. 237, June 30, 1972, pp. 510-512.

4. A. F. Lann, M. Golosovsky, D. Davidov, and A. Frankel, "Mapping the thickness of conducting layers by a mm-wave near-field microscope," 1998 IEEE MTT-S Int. Microwave Symp. Dig., Baltimore, MD, June 7-12, 1998, pp. 1337-1340.

5. R. J. Gutmann, J. M. Borrego, P. Chakrabarti, and M.-S. Wang, "Microwave scanning microscopy for planar structure diagnostics," 1987 IEEE MTT-S Int. Microwave Symp. Dig., 1987, pp. 281-284.

6. C. P. Vlahacos, R. C. Black, S. M. Anlage, A. Amar, and F. C. Wellstood, "Near-field scanning microwave microscope with $100 \mu \mathrm{m}$ resolution," Appl. Phys. Lett., 69(21), Nov. 18, 1996, pp. 32723274.

7. B. J. Feenstra, C. P. Vlahacos, A. S. Thanawalla, D. E. Steinhauer, S. K. Dutta, F. C. Wellstood, and S. M. Anlage, "Near-field scanning microwave microscopy: measuring local microwave properties and electric field distributions," 1998 IEEE MTT-S Int. Microwave Symp. Dig., Baltimore, MD, June 7-12, 1998, pp. 965-968.

8. T. Wei, X.-D. Xiang, W. G. WallaceFreedman, and P. G. Schultz, "Scanning tip microwave near-field microscope," Appl. Phys. Lett., 68(24), June 10, 1996, pp. 3506-3508.

9. M. Tabib-Azar, D.-P. Su, S. LeClair, and G. Ponchak, "The ultimate resolution of superresolution imaging using evanescent microwave probes," To appear in Review of Scientific Instruments.

10. R. N. Simons, G. E. Ponchak, K. S. Martzaklis, and R. R. Romanofsky, "Channelized coplanar waveguide: discontinuities, junctions, and propagation characteristics," 1989 IEEE MTT-S Int. Microwave Symp. Dig., Long Beach, CA, June 1315, 1989, pp. 915-918. 